\title{
Morfometría de los Ramos Nerviosos para los Músculos Interóseos Plantares del Pie en el Hombre
}

\author{
Morphometry of Nerve Branches for Plantar Interossei Muscles in the Foot of Man \\ "Bélgica Vásquez; **Mariano del Sol \& *** J. C. Coronado
}

VÁSQUEZ, B.; DEL SOL, M. \& CORONADO, J. C. Morfometría de los ramos nerviosos para los músculos interóseos plantares del pie en el Hombre. Int. J. Morphol., 29(1):80-83, 2011.

RESUMEN: La inervación del pie ha requerido especial atención por su utilidad en el abordaje clínico y quirúrgico de patologías y síndromes dolorosos como talalgias, fascitis plantar y fibromatosis plantar, entre otras. Estudios realizados, han permitido conocer la importancia funcional de los músculos interóseos plantares. Sin embargo, no se conoce mayores detalles de su inervación. Realizamos un estudio morfométrico en 5 muestras de ramos nerviosos para el primer, segundo y tercer músculo interóseo en cadáveres de individuos adultos, masculinos. Se obtuvieron secciones transversales de cada uno de los nervios después de su origen, a nivel del plano profundo de la región plantar del pie. Realizamos cortes de $4 \mu \mathrm{m}$ de grosor los cuales fueron teñidos con Hematoxilina-Eosina (H.E.). El promedio de fascículos en el ramo para el primer músculo interóseo (R1 IOP) fue 1 (D.E. 0,00); para el segundo músculo interóseo (R2 IOP) fue 2 (D.E. 0,45) y para el tercer músculo interóseo (R3 IOP) fue 2 (D.E. 1,00). El promedio de fibras para el R1 IOP, R2 IOP Y R3 IOP fue: 547 (D.E. 153), 644 (D.E. 258) y 1.161 (D.E. 465) respectivamente. El área promedio del R1 IOP, R2 IOP Y R3 IOP fue: 0,06 $\mathrm{mm}^{2}$ (D. E. 0,02), 0,08 $\mathrm{mm}^{2}$ (D. E. 0,03), 0,14 $\mathrm{mm}^{2}$ (D. E. 0,06), respectivamente. El estudio morfométrico y estereológico de estos ramos nerviosos espera aportar al conocimiento de los profesionales que deben intervenir clínica y quirúrgicamente en la región plantar del pie.

PALABRAS CLAVE: Músculos interóseos plantares; Inervación y Morfometría.

\section{INTRODUCCIÓN}

El conocimiento de la inervación del pie es importante por su utilidad en el abordaje clínico de diversas patologías y en reconstrucciones microquirúrgicas del pie y la mano (Placer \& Lozano, 2007). Así, alteraciones morfológicas y funcionales del pie, tales como dedo en martillo o en garra, pueden ser causadas por debilidad de músculos del plano profundo del pie asociados a lesiones de los ramos que los inervan (Bisschop et al., 1979; Núñez-Samper \& Llanos, 2007; Izquierdo, 2009).

La inervación de los músculos interóseos está asociada a fibras provenientes del ramo profundo del nervio plantar lateral, hecho que ha sido señalado por numerosos autores sin mayores detalles en su descripción (Rouvière \& Delmas, 1984; Benninghof \& Goerttler, 1986; del Sol \& Prates, 1991; Latarjet \& Ruiz Liard, 2005; Moore \& Dalley,
2007). Por su parte, Schäfer \& Symington (1909); Chatain \& Bustamante, 1986 y Williams et al., 1995 indicaron que ocasionalmente el ramo profundo del nervio plantar lateral no inerva los músculos interóseos del cuarto espacio, si no que serían inervados por el ramo superficial del nervio plantar lateral.

del Sol et al. (1992), determinaron el sitio de división del nervio plantar lateral en ramos superficial y profundo en relación a la longitud total del pie, dividiéndolo en seis segmentos. Estos autores encontraron que en el $96 \%$ de los casos se dividía en la mitad posterior del tercio medio del pie y el porcentaje restante lo hacía en la mitad anterior del tercio medio del pie. Posteriormente, del Sol et al. (1996), en un estudio realizado en 100 pies, encontraron que el ramo profundo del nervio plantar lateral da una doble inervación al músculo aductor del hálux.

\footnotetext{
* Facultad de Ciencias de la Salud, Universidad Autónoma de Chile, Sede Temuco, Chile.

** Universidad de La Frontera, Temuco, Chile.

**** Universidad Mayor, Sede Temuco, Chile.

Financiado por DIUFRO, Proyecto No 120625, Universidad de La Frontera, Chile.
} 
En estudios morfométricos de los nervios tibial, plantar medial y plantar lateral, realizados por del Sol et al. (2005), determinaron que el nervio plantar lateral, a nivel de su origen, tenía en promedio 14,8 (D.E. 3,96) fascículos; cuya área promedio correspondió a $1,48 \mathrm{~mm}^{2}$ y un número promedio de fibras de 11.679 (D.E. 1.710). Este nervio, en su trayectoria hacia el pie, antes de su división en ramos superficial y profundo, inerva los músculos abductor del dedo mínimo y cuadrado plantar, dando además, el ramo sensitivo cutáneo lateral que se dirige al margen lateral del pie. Del Sol et al. (2006), describieron el número de fascículos, área y número de fibras que inervan a estos músculos.

Conocer parámetros morfométricos de los ramos que inervan a los músculos interóseos del pie, adquiere importancia dado que no existe en la literatura trabajos específicos al respecto. Por otra parte, determinar aspectos como: número de fascículos, área y número de fibras que inervan a estos músculos proporciona gran ayuda durante los procedimientos quirúrgicos de la musculatura intrínseca del pie, como también en las estimulaciones con propósitos terapéuticos. Así, el objetivo de esta investigación fue determinar el número de fascículos, área y número de fibras que inervan el primero, segundo y tercer músculos interóseos.

\section{MATERIAL Y MÉTODO}

Realizamos un estudio morfométrico en 5 muestras de ramos nerviosos para el primero, segundo y tercer músculos interóseos, en cadáveres de individuos adultos, masculinos, provenientes de la Facultad de Medicina de la Uni- versidad de La Frontera, Temuco, Chile. Se obtuvieron secciones transversales de cada uno de los nervios después de su origen, a nivel del plano profundo de la región plantar del pie, los cuales, fueron procesados para su inclusión en paraplast. Cortes de $4 \mu \mathrm{m}$ de grosor, fueron montados en portaobjetos y teñidos con H.E. En cada muestra, determinamos, por planimetría a través del conteo de puntos, el número de fascículos existentes en cada nervio, el número de fibras nerviosas de cada fascículo y el área de los fascículos que constituían cada nervio. Los cortes fueron fotografiados utilizando un microscopio Carl Zeiss, Axiolab, con cámara MC 80 DX. Para la morfometría, utilizamos un microscopio Olympus con retículo ocular (1 mm/0,01 mm) calibrándolo con un retículo objetivo (Mandarim de Lacerda, 1995).

\section{RESULTADOS}

El promedio de fascículos en las 5 muestras estudiadas en el ramo para el primer músculo interóseo (R1 IOP) fue 1 (D.E. 0,00); para el segundo músculo interóseo (R2 IOP) fue 2 (D.E. 0,45) y para el tercer músculo interóseo (R3 IOP) fue 2 (D.E. 1,00). El número total de fibras para el R1 IOP, R2 IOP Y R3 IOP se presenta en la Fig. 1.

El promedio de fibras para el R1 IOP, R2 IOP Y R3 IOP fue: 547 (D.E. 153), 644 (D.E. 258) y 1.161 (D.E. 465) respectivamente.

En la Tabla I se muestra la suma de las áreas de los fascículos que constituyen los ramos para el primero, segundo y tercer músculo interóseo.

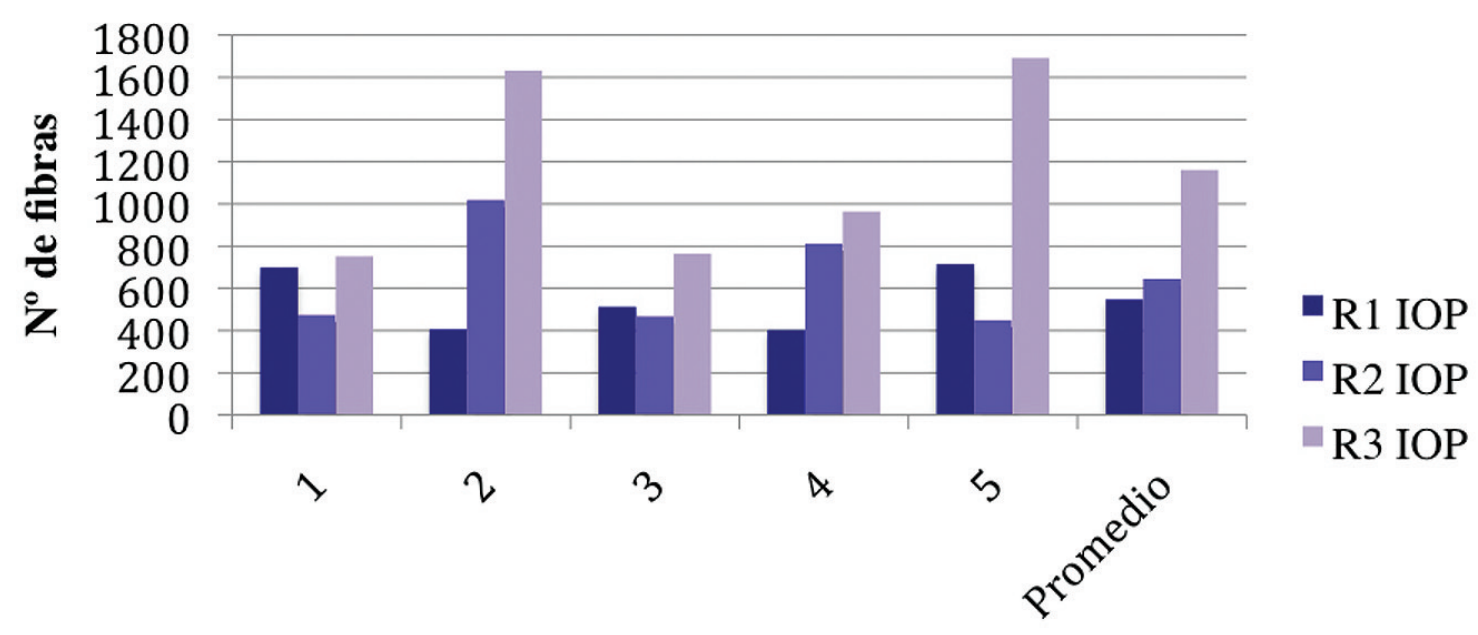

\section{$\mathbf{N}^{0}$ de muestras}

Fig. 1. Número de fibras en cada uno de los ramos para el primero (R1 IOP), segundo (R2 IOP) y tercer músculo interóseo (R3 IOP). 
Tabla I. Suma de las áreas de los fascículos para el primer músculo interóseo (R1 IOP) para el segundo músculo interóseo (R2 IOP) y para el tercer músculo interóseo (R3 IOP).

\begin{tabular}{lccc} 
& \multicolumn{3}{c}{ Área en $\mathrm{mm}^{2}$} \\
\hline $\mathrm{n}^{\mathbf{0}}$ muestra & $\mathrm{R}$ 1 IOP & $\mathrm{R} 2 \mathrm{IOP}$ & $\mathrm{R} 3 \mathrm{IOP}$ \\
\hline 1 & 0,09 & 0,06 & 0,09 \\
2 & 0,05 & 0,12 & 0,21 \\
3 & 0,06 & 0,06 & 0,09 \\
4 & 0,04 & 0,10 & 0,11 \\
5 & 0,07 & 0,06 & 0,20 \\
\hline Promedio & 0,06 & 0,08 & 0,14 \\
D.E. & 0,02 & 0,03 & 0,06 \\
\hline
\end{tabular}

\section{DISCUSIÓN}

Los autores de la anatomía clásica concuerdan en que los músculos interóseos del pie son inervados por el ramo profundo del nervio plantar lateral (Rouvière \& Delmas; Benninghof \& Goerttler; Chatain \& Bustamante; del Sol \& Prates, 1991; Latarjet \& Ruiz Liard; Moore \& Dalley). Sin embargo, carecen de descripciones detalladas respecto a estudios mesoscópicos y morfométricos como los son el número de fascículos y fibras que inervan dichas estructuras. Esta falta de información, se hace más evidente cuando se desean realizar procedimientos quirúrgicos de reparación por patologías propias del pie o cirugías reconstructivas de éste por daño catastrófico. Por otro lado, las investigaciones más recientes apuntan, principalmente, a describir con más detalle la vascularización existente en la región anterior del pie que sus características de inervación específica (Gabrielli et al., 2001).

Según Izquierdo (2009), en pacientes operados por deformidades óseas y articulares de los dedos del pie, la obtención de un resultado aceptable no solo depende del tipo de cirugía, si no también de las diferentes posibilidades técnicas del abordajes. En este sentido, será importante el conocimiento de la inervación específica de los músculos implicados y sus características morfométricas. Por lo anterior, el conocimiento del número de fibras que constituyen los nervios que inervan a los músculos interóseos, toma importancia, ya que, son estructuras que podrían estar involucradas en la etiología de las deformidades del pie. Resulta relevante, saber que el tercer músculo interóseo plantar recibe el mayor número de fibras, hecho que se justifica por el tamaño de este músculo, el cual posee características distintas a los otros y a su relación que mantiene con el eje del pie.

Por otro lado, en el abordaje quirúrgico, conocer aspectos relacionado con la trayectoria y/o relaciones anatómicas de los ramos que inervan estos músculos, resulta importante. Coronado \& del Sol (2010) señalaron, que los ramos musculares para los músculos interóseos deben cruzar inferiormente la arteria plantar lateral; situación que indica una alta probabilidad de lesión ante una daño vascular en la región plantar media del pie.

Debido a los escasos estudios en relación a la inervación de los músculos interóseos plantares esta investigación aporta datos cuantitativos que contribuyen al conocimiento anatómico macroscópico y morfométrico.

VÁSQUEZ, B.; DEL SOL, M. \& CORONADO, J. C. Morphometry of nerve branches for plantar interossei muscles in the foot of man. Int. J. Morphol., 29(1):80-83, 2011.

SUMMARY: Innervation of the foot has required special attention in view of its use in clinical and surgical treatment of painful diseases and syndromes such as talalgia, plantar fasciitis and plantar fibromatosis are among those mentioned. Studies realized have made possible the functional significance of plantar interossei muscles. However, there is no information regarding greater detail of their innervation. A morphometric study was carried out in 5 nerve branches for the first, second and third plantar interossei muscles in cadavers of adult male individuals. Cross sections were obtained of each one of the nerves after their origin, at the deep level of the plantar region of the foot. Cuts of $4 \mu \mathrm{m}$ thick were realized which were stained with Hematoxylin-Eosin (H.E.). Average fascicles in the branch for the first interosseous muscle (R1 IOP) was 1 (D.E. 0,00); for the second interosseous muscle (R2 IOP) was 2 (D.E. 0.45); and for the third interosseous muscle (R3 IOP) was 2 (D.E. 1.00). The average of fibers for the 1 R1 IOP, R2 IOP was: 547 (D.E. 153), 644 (D.E. 258) and 1.161 (D.E. 465) respectively. The average area of R1 IOP, R2 IOP Y R3 IOP was: $0.06 \mathrm{~mm}^{2}$ (D. E. 0.02 ), $0.08 \mathrm{~mm}^{2}$ (D. E. 0.03 ), $0.14 \mathrm{~mm}^{2}$ (D. E. 0.06), respectively. The morphometric and stereological study of these nerve branches is expected to contribute to the information available to professionals who must clinically and surgically treat the plantar region of the foot.

KEY WORDS: Plantar interossei muscles; Innervation and Morphometry. 


\section{REFERENCIAS BIBLIOGRÁFICAS}

Benninghof, A. \& Goerttler, K. Trattado di anatomía funzionale. $2^{\mathrm{a}}$ ed. Padova, Paccin, 1986, V3.

Bisschop, G.; Claparede, P.; Bence, Y.; Commandre, F. \& Dumoulin, J. Exploration neuromusculaire du pied: interet des examenes electromyographiquies et neurographiques. Electrodiagnostic therapie revue européene delectrophysiologie applique, 16(2),1979.

Chatain, I. \& Bustamante, J. Anatomía macroscópica y funcional. México, Iberoamericana, 1986.

Coronado, J. C. \& del Sol, M. Inervación de los músculos interóseos plantares del pie humano. Int. J. Morphol., 28(4):999-1002, 2010.

del sol, M. \& Prates, J.C. Ramos componentes de la división del nervio plantar lateral en el hombre. Rev. Chiv. Anat. 9(2):107-11, 1991.

del Sol, M.; Prates, J.C. \& Ribeiro, E. Local de divisão do nervo plantar lateral. Rev. Bras. Cir. 82(1): 11-3, 1992.

del Sol, M; Prates, J.C. ; Olave, E.; Mandiola, E. \& Gabrielli, C. Inervación del músculo abductor de hálux. Rev. Chzil. Anat., 14(1):73-8, 1996.

del Sol, M.; Vasconcellos, A.; Parra, R. \& Vásquez, B. Morfometría de los nervios tibial y plantares. Int. J. Morphol., 23(4):399- 404, 2005.

del Sol, M.; Vásquez, B. \& Nicklas, C. Morfometría de los ramos del nervio plantar lateral antes de su división en ramos superficial y profundo. Int. J. Morphol., 24(1):191-194, 2006.

Gabrielli, C.; Braga, T. M. T.; Olave, E.; Froehner Jr. I. \& Rodrigues, K. I. Aspectos anatómicos y biométricos de la arteria dorsal del pie en humanos. Rev. Chil. Anat., 19(3):325-30, 2001

Izquierdo, J. Tratamiento quirúrgicos de las deformidades digitales de los pies. Artroplastía versus cirugía mínimamente invasiva. Rev. Int. Cien. Pod. 3(2):7$25,2009$.

Latarjet, M. \& Ruiz Liard, A. Anatomía humana. $4^{\mathrm{a}} \mathrm{ed}$. Buenos Aires, Panamericana, 2005.
Mandarim-de-Lacerda, C. A. Métodos quantitativos em morfologia. Rio de Janeiro, EDUERJ, 1995.

Moore, K. L. \& Dalley, A. F. Anatomía con orientación clínica. $5^{\mathrm{a}}$. ed. Buenos Aires, Panamericana, 2007.

Núñez-Samper, M \& Llanos, L. Biomecánica, Medicina y Cirugía del pie. $2^{\mathrm{a}}$ ed. Barcelona, Masson, 2007.

Placer, A. \& Lozano J. A. Transferencia microquirúrgica del $2^{\circ}$ dedo del pie para reconstrucción de mano catastrófica. An. Sist. Sanit. Navar., 30 (3): 481-486, 2007.

Rouvière, H. \& Delmas, A. Anatomie humaine. $12^{\mathrm{a}}$ ed. Paris, Masson, 1984.

Schäfer, E. A. \& Symington, J. Quain's elements of anatomy. $11^{\mathrm{a}}$ ed. London, Logmans, Green and Co., 1909. V3.

Williams, P.; Warwick, R.; Dyson, M. \& Bannister, L. Gray Anatomia. $37^{\mathrm{a}}$ ed. Rio de Janeiro, Guanabara Koogan, 1995.

Dirección para correspondencia:

Prof. Dr. Mariano del Sol

Facultad de Medicina

Universidad de La Frontera

Casilla 54-D

Temuco

CHILE

Email: mdelsol@ufro.cl

Recibido : 09-09-2010

Aceptado: 01-12-2010 